\title{
Osteoclast regulation of osteoblasts via RANK-RANKL reverse signal transduction in vitro
}

\author{
SHIQIAN ZHANG ${ }^{1}$, XIAOYU WANG ${ }^{1}$, GUOJUN LI $^{1}$, YANG CHONG ${ }^{1}$, \\ JIE ZHANG ${ }^{1}$, XINZHEN GUO $^{1},{\text { BAOXIN } \mathrm{LI}^{2} \text { and ZHENGGANG BI }}^{1}$ \\ ${ }^{1}$ Department of Orthopedic Surgery, The First Affiliated Hospital of Harbin Medical University, Harbin, Heilongjiang 150001; \\ ${ }^{2}$ Department of Pharmacology, Harbin Medical University, Harbin, Heilongjiang 150081, P.R. China
}

Received February 26, 2016; Accepted May 11, 2017

DOI: $10.3892 / \mathrm{mmr} .2017 .7039$

\begin{abstract}
The treatment of osteoporosis typically inhibits the activity of osteoclasts, which subsequently results in the suppression of bone formation and maintenance, however the underlying mechanism remains to be elucidated. The receptor activator of nuclear factor $\kappa$-B ligand (RANKL)-receptor activator of nuclear factor $\kappa-B$ (RANK) signaling axis is important in the osteoblast regulation of osteoclasts. RANKL surface-bound molecules expressed on $\mathrm{T}$ cells stimulate a reverse signaling transduction in order to regulate the $\mathrm{T}$ cells, therefore the present study hypothesized that RANKL expressed on osteoblasts may transfer reverse signals to regulate osteoblasts. A series of experiments were designed to test the hypothesis, using MTT, stealth RNA interference, reverse transcription-quantitative polymerase chain reaction, western blot analysis, alkaline phosphatase activity assay and alizarin red staining. The present study observed the role of RANK-RANKL reverse signaling on osteoblasts, regulated by osteoclasts. Osteoblasts were treated with recombinant RANK proteins. The soluble RANK enhanced the mineralization of osteoblasts. When the RANKL was knocked down in the osteoblast, RANK demonstrated a weak osteogenic effect on the RANKL-deficient osteoblast compared with the wild-type osteoblast which served as a control. Addition of soluble RANK activated the p38 mitogen activated protein kinase (MAPK) signaling pathway in the osteoblast and blocking this same pathway in E1 cells reduced the effect of RANK. In the co-culture system of osteoblasts and osteoclasts, p38 MAPK in E1 cells was phosphorylated a short time following co-culture and the phosphorylation then blocked by abundant soluble RANKL. The findings suggested that RANKL expressed on
\end{abstract}

Correspondence to: Professor Zhenggang $\mathrm{Bi}$, Department of Orthopedic Surgery, The First Affiliated Hospital of Harbin Medical University, 23 Youzheng Street, Harbin, Heilongjiang 150001, P.R. China

E-mail: drbizhenggang@163.com

Key words: osteoporosis, receptor activator of nuclear factor $\kappa-\mathrm{B}$ ligand-receptor activator of nuclear factor $\kappa-\mathrm{B}$, osteoclast, osteoblast, reverse signal osteoblasts transferred reverse signals from the exterior of the cell to the interior, which regulated the osteoblasts.

\section{Introduction}

The functional activity of osteoblasts and osteoclasts is synergistic and an imbalance may result in a variety of diseases, including osteoporosis. Osteoblasts regulate osteoclasts via the receptor activator of nuclear factor $\kappa-\mathrm{B}$ ligand (RANKL)-receptor activator of nuclear factor $\kappa-B$ (RANK) signaling pathway. RANKL is expressed on osteoblasts and $\mathrm{T}$ cells. It binds the receptor RANK, which is produced on osteoclasts and their progenitors. The interaction of RANK with RANKL is required for osteoclast formation, differentiation, activation and survival. RANKL-RANK signaling is important in the osteoblast regulation of osteoclasts (1). Osteoprotegerin (OPG) and soluble RANK have been developed as pharmaceutical candidates (2), and anti-human RANKL neutralizing antibody (denosumab) has gained approval from the Food and Drug Administration and the European Medicines Agency for the treatment of postmenopausal osteoporosis and cancer-associated bone disorders (3).

RANKL is a member of the tumor necrosis factor (TNF) super-family and is produced on osteoblasts and $\mathrm{T}$ cells. It has previously been suggested that this membrane-integrated TNF ligand may receive signals, subsequently acting as a receptor, to transmit positive and negative feedback signals into the ligand-bearing cell (4). Therefore, reverse signaling enables a two-way communication in cell-to-cell signaling, and it is conceivable that this bidirectional signal exchange contributes to the plasticity of the ligand-receptor systems. It has been reported that RANKL expressed on the surface of activated cluster of differentiation (CD)4+ T helper (Th)1 cells is involved in a reverse signaling process to suppress interferon $-\gamma$ secretion via activated Th1 cells. The process is associated with the activation of p38 mitogen-activated protein kinase (MAPK) and is blocked by SB203580, a p38 MAPK specific inhibitor (5). Recombinant RANK may upregulate interleukin-8 (IL-8) in B chronic lymphocytic leukemia cells via this reverse signaling process (6).

It has additionally been reported that bone formation may be regulated by osteoclast activity. The bone remodeling process involves a balance between bone resorption and formation, therefore this equilibrium may be controlled via 
a concerted action between the two cell types. However, the mechanism by which osteoclast feedback regulates osteoblasts remains to be elucidated. Previous research has indicated that the trans-membrane RANKL expressed on $\mathrm{T}$ cells regulates their activity via reverse signaling (4). Therefore, the present study hypothesized that the RANKL expressed on osteoblasts may receive reverse transduced signals and result in osteoclast regulation of osteoblasts via RANK-RANKL reverse signaling. This novel, identified pathway may contribute to the further understanding of how osteoclasts regulate bone formation. To test the hypotheses, the present study investigated the effects of recombinant RANK protein on osteoblast differentiation/mineralization in MC3T3-E1 (E1) cells and investigated the mechanism and signaling pathways of RANK-induced osteoblastogenesis. It was observed that RANKL exhibited a reverse signal transduction ability and this finding may aid in the identification of the specific underlying mechanism that regulates the coupling between bone formation and resorption.

\section{Materials and methods}

Reagents. Recombinant soluble RANK (sRANK) and recombinant soluble RANKL (sRANKL) were prepared by $E$. coli as previously described (7). Various single-amino-acidmutant RANK sequences were designed based on the RANKL-RANK structure (8), and their binding affinity to RANKL was assessed. A mutant-RANK (Trp81Ala) with an extremely low binding affinity to RANKL was identified and subsequently termed mu-RANK. Mu-RANK exhibited a single amino acid difference compared with the wild-type RANK and was used as a control. The binding affinity of the Glu225Ala mutant RANKL, is dramatically decreased by 100 -fold and this mutant RANKL (mu-RANKL) was used as a control in the test. SB203580 p38 inhibitor was purchased from Sigma-Aldrich; Merck KGaA (Darmstadt, Germany). $\beta$-glycerophosphate, ascorbic acid phosphate and dexamethasone (Dex) were additionally purchased from Sigma-Aldrich; Merck KGaA.

Cell culture. The MC3T3-E1 (E1) and RAW246.7 mouse pre-osteoblastic cells were provided by The Cell Bank Type Culture Collection, Chinese Academy of Sciences (Shanghai China). The cells were incubated with $\alpha$-Minimal Essential Medium (MEM) (HyClone; GE Healthcare Life Sciences, Logan, UT, USA) supplemented with $10 \%$ fetal bovine serum (FBS; Gibco; Thermo Fisher Scientific, Inc., Waltham, MA, USA), $100 \mathrm{U} / \mathrm{ml}$ penicillin and $100 \mu \mathrm{g} / \mathrm{ml}$ streptomycin (Sigma-Aldrich; Merck KGaA) at $37^{\circ} \mathrm{C}$ in a humidified atmosphere containing $5 \% \mathrm{CO}_{2}$. At $\sim 80 \%$ confluence, the E1 cells were separated and cultured in complete medium containing osteogenic factors (0.05 mM L-ascorbic acid, $100 \mathrm{nM}$ Dex and $10 \mathrm{mM} \beta$-glycerophosphate). Co-culture was initiated when the RAW246.7 cells were plated at a density of $3 \times 10^{5}$ cells per well on the formed E1 cell layer, following osteogenic differentiation.

Stealth RNA interference (RNAi). Stealth RNAs targeting the 25-nucleotide sequence of RANKL and the control were obtained from Thermo Fisher Scientific, Inc. The primers sequences were as follows: Forward, 5'-GCGCAGAUGGAU CCUAACAdTdT-3' and reverse, 5'-UGUUAG GAUCCA
UCUGCGCdTdT-3' for siRNA and forward, 5'-UUCUCC GAACGUGUCACGUTT-3' and reverse, 5'-ACGUGACAC GUUCGGAGAATT-3' for negative control. Before transfection, cells were plated in six-well culture dishes at a density of $1 \mathrm{c} 10^{5}$ cells per dish. When the confluence reached $70 \%$, E1 cells were transfected with $10 \mathrm{nM}$ stealth RNAs using Lipofectamine $^{\mathrm{TM}}$ (Thermo Fisher Scientific, Inc.) according to the manufacturer's protocol.

Experimental design. The study consisted of four subinvestigations on differing groups: i) E1 cells maintained with $10^{-6}, 10^{-5}$ and $10^{-4} \mathrm{~g} / 1 \mathrm{RANK}$ protein (9); ii) E1 cells transfected with $10 \mathrm{nM}$ stealth RNA in the presence of $10^{-4} \mathrm{~g} / 1$ RANK protein; iii) E1 cells in the presence of $10^{-4} \mathrm{~g} / \mathrm{l}$ RANK protein with or without 2-10 $\mu \mathrm{M}$ p38 inhibitor (SB203580) and iv) E1 cells co-cultured with RAW 246.7 cells with 5 nM sRANKL.

MTT assay. To investigate the effects of sRANK on cell growth, E1 cells were inoculated at $5 \times 10^{3}$ cells per well in 96-well culture plates. Following a 24 h culture period, cells were then treated with $10^{-6}, 10^{-5}$ and $10^{-4} \mathrm{~g} / 1$ of sRANK or without sRANK as a control for 24,48 and $72 \mathrm{~h}$. The cells were washed twice with PBS. A total of $200 \mu 1$ complete medium with supplement $20 \mu 15 \mathrm{mg} / \mathrm{ml}$ MTT solution (Sigma-Aldrich; Merck KGaA) was added to each well. Following incubation at $37^{\circ} \mathrm{C}$ for $4 \mathrm{~h}$, the medium was replaced with $200 \mu$ l dimethyl sulfoxide (Sigma-Aldrich; Merck KGaA) and vibrated for $15 \mathrm{~min}$. Finally, the absorbance was measured at a wavelength of $490 \mathrm{~nm}$ by a microplate spectrophotometer. All experiments were performed in triplicate.

Alkaline phosphatase activity assay (ALP) and alizarin red staining. The ALP activity assay was conducted using an ALP kit (Nanjing Jiancheng Bioengineering Institute, Nanjing, China), according to the manufacturer's protocol. Alizarin red staining was performed to detect calcium deposits. The cultured cells were fixed by $10 \%$ neutral buffered formalin and stained with $1 \%$ alizarin red $\mathrm{S}$ solution for $10 \mathrm{~min}$ at room temperature. The calcified nodules were observed under a light microscope (Olympus Corporation, Tokyo, Japan).

Western blot analysis. Cells were lysed on ice for 5 min using radioimmunoprecipitation assay buffer (Beyotime Institute of Biotechnology, Beijing, China), and were then sonicated briefly and centrifuged for $10 \mathrm{~min}$ at $14,000 \mathrm{x} \mathrm{g}$ in a cold microfuge. Protein concentrations were determined using a bicinchoninic acis assay (Beyotime Institute of Biotechnology). Total protein samples (40 $\mu \mathrm{g}$ per lane) were separated by $10 \%$ SDS-PAGE and transferred to nitrocellulose membranes (EMD Millipore, Billerica, MA, USA). Following blocking with 5\% dried skim milk in TBS/0.05\% Tween-20 (TBST) for $1 \mathrm{~h}$ at room temperature, the membranes were incubated overnight at $4^{\circ} \mathrm{C}$ with primary antibodies against osteocalcin (catalog no. sc-30045; 1:1,000 dilution; Santa Cruz Biotechnology, Inc., Dallas, TX, USA), p38 (catalog no. sc-7149; 1:1,000 dilution; Santa Cruz Biotechnology, Inc.), phospho-p38 (catalog no. 4092; 1:1,000 dilution; Cell Signaling Technology, Inc. Danvers, MA, USA), and $\beta$-actin (catalog no. NB600-503; 1:5,000 dilution; Novus Biologicals, LLC. Littleton, CO, USA) in TBST. Following 3 washes with TBST, membranes were incubated 


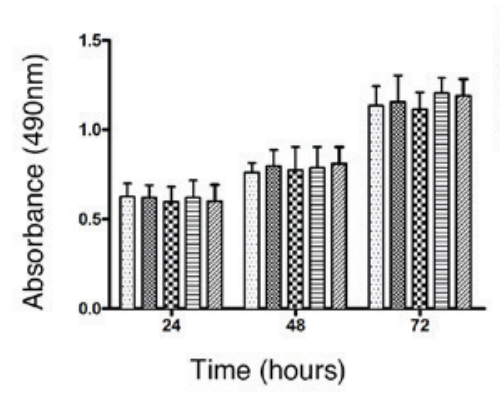

C

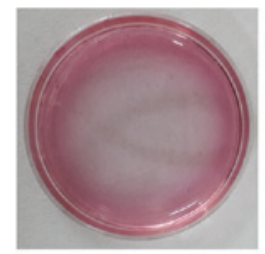

Mu-RANK

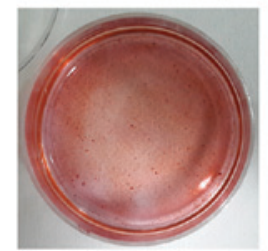

$10^{-5} \mathrm{~g} / \mathrm{L}$

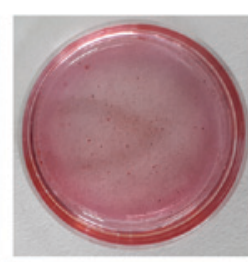

$10^{-6} \mathrm{~g} / \mathrm{L}$

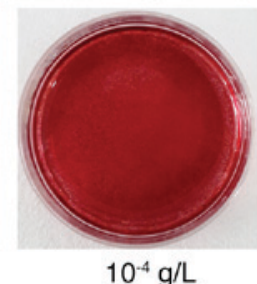

B

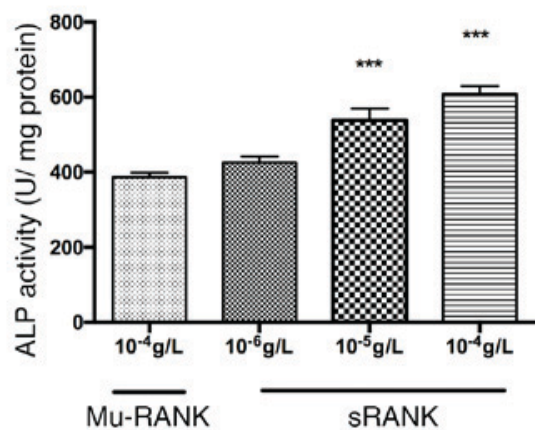

D

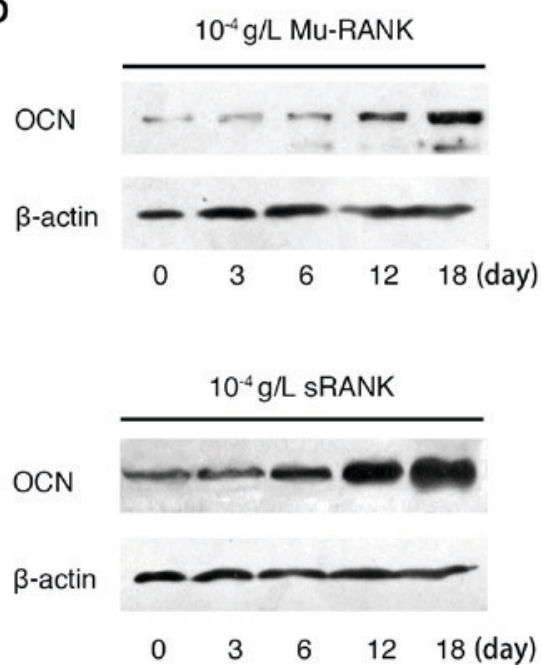

Figure 1. In-vitro analysis of osteoblastogenesis effect of RANK protein. (A) The proliferation activity of E1 cells following exposure to Mu-RANK (10 $\left.0^{-4} \mathrm{~g} / \mathrm{l}\right)$ and sRANK $\left(10^{-6}, 10^{-5}\right.$ and $\left.10^{-4} \mathrm{~g} / \mathrm{l}\right)$ evaluated by MTT assay. (B) Effects of sRANK on ALP activity in mouse pre-osteoblastic cells. ALP activity was expressed as the mean \pm standard deviation. ${ }^{* * * *} \mathrm{P}<0.001$ vs. control. (C) Mineralization of E1 cells was evaluated by alizarin red staining. (D) The expression of OCN following Mu-RANK $\left(10^{-4} \mathrm{~g} / \mathrm{l}\right)$ and sRANK $\left(10^{-4} \mathrm{~g} / \mathrm{l}\right)$ treatment, evaluated by western blotting. RANK, receptor activator of nuclear factor $\mathrm{\kappa}-\mathrm{B}$; OCN, osteocalcin; ALP, alkaline phosphatase activity assay; sRANK, recombinant soluble RANK; Mu-RANK, mutant RANK.

with horseradish peroxidase-conjugated anti-rabbit immunoglobulin (Ig)G antibody (catalog no. A0208; 1:5,000 dilution; Beyotime Institute of Biotechnology) for $1 \mathrm{~h}$ at room temperature. Protein expression signals were detected with Enhanced Chemiluminescence-plus substrate (Applygen Technologies, Inc., Beijing, China) using the ChemiDoc ${ }^{\mathrm{TM}} \mathrm{XRS}+$ system (Bio-Rad Laboratories, Inc. Hercules. CA, USA), according to the manufacturer's protocol.

Reverse transcription-quantitative polymerase chain reaction $(R T-q P C R)$. Total RNA of the cells was isolated using TRIzol ${ }^{\circledR}$ (Invitrogen; Thermo Fisher Scientific, Inc.). To carry out qPCR, cDNAs were synthesized from $1 \mu \mathrm{g}$ of the RNA using Transcriptor First Strand cDNA Synthesis kit (Roche Diagnostics $\mathrm{GmbH}$, Mannheim, Germany), according to the manufacturer's protocol. qPCR analysis of RANKL and GAPDH was conducted using the ABI Prism 7300 real-time PCR system (Applied Biosystems; Thermo Fisher Scientific, Inc.) and PowerUp ${ }^{\mathrm{TM}}$ $\mathrm{SYBR}^{\mathrm{TM}}$ Green Master mix (Applied Biosystems; Thermo Fisher Scientific, Inc.). Each reaction mixture contained $10 \mu \mathrm{l}$ SYBR Green master mix, $7 \mu 1$ RNase-free $\mathrm{H}_{2} \mathrm{O}, 1 \mu 110 \mathrm{mM}$ forward primer, $1 \mu 110 \mathrm{mM}$ reverse primer, and $1 \mu \mathrm{l} \mathrm{cDNA}$ in a final reaction volume of $20 \mu \mathrm{l}$. Cycling conditions were as follows: An initial denaturation at $95^{\circ} \mathrm{C}$ for $10 \mathrm{~min}$, followed by
40 cycles of denaturation at $95^{\circ} \mathrm{C}$ for $15 \mathrm{sec}$, annealing at $55^{\circ} \mathrm{C}$ for $15 \mathrm{sec}$ and elongation at $72^{\circ} \mathrm{C}$ for $15 \mathrm{sec}$. Gene expression was normalized to the internal reference gene GAPDH. The $2^{-\Delta \Delta C q}$ method (10) was used to determine relative fold changes. The primers were designed as follows: Forward, 5'-GGCAAG CCTGAGGCCCAGCCATTT-3', and reverse, 5'-GTCTCAGTC TATGTCCTGAACTTT-3' for RANKL and forward, 5'-CAC CATGGAGAAGGCCGGGG-3', and reverse, 5'-GACGGA CACATTGGGGGTAG-3' for GAPDH. All experiments were performed in triplicate.

Statistical analysis. Statistical analysis was performed using SPSS software, version 17.0 (SPSS, Inc., Chicago, IL, USA). The data are expressed as the mean \pm standard deviation from all experiments. Significant differences were determined using one-way ANOVA with Dunnett's post-hoc test or unpaired Student's t-test. $\mathrm{P}<0.05$ was considered to indicate a statistically significant difference.

\section{Results}

RANK protein increases osteoblastogenesis in El cells. The proliferation activity of E1 cells was examined via MTT assay following sRANK $\left(10^{-6}, 10^{-5}\right.$ and $\left.10^{-4} \mathrm{~g} / \mathrm{l}\right)$ treatment for 24,48 
A

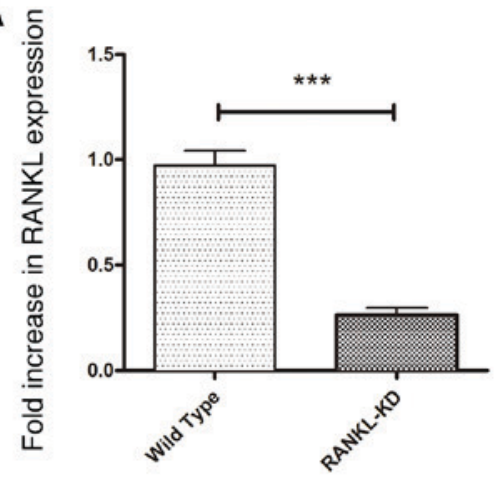

B

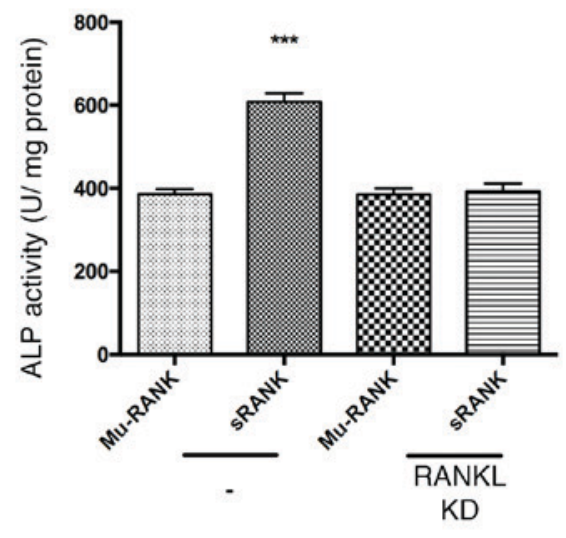

C
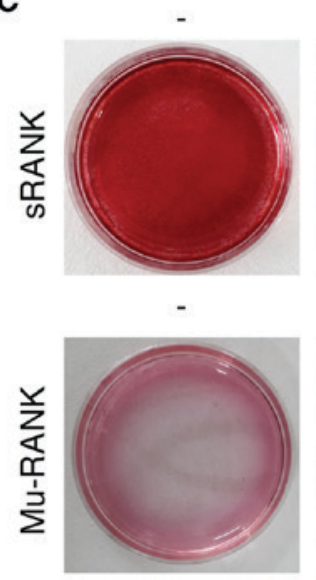

RANKL KD

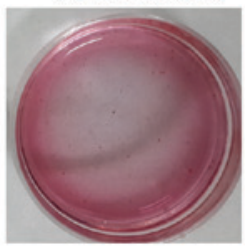

RANKL KD

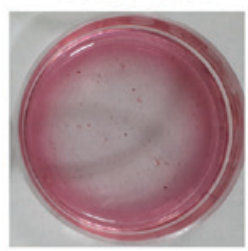

Figure 2. Signaling via RANKL is involved, in part, in RANK-induced osteoblast differentiation. (A) Expression of RANKL was measured by reverse transcription-polymerase chain reaction. RANKL gene expression was suppressed by small interfering RNA-mediated knockdown with 10 nM stealth RNAi in E1 cells. (B) Treated cells were further cultured with $10^{-4} \mathrm{~g} / 1 \mathrm{sRANK}$ protein and ALP activity was measured. Data was expressed as the mean \pm standard deviation. ${ }^{* * *} \mathrm{P}<0.001$ vs. each control. (C) Mineralization of E1 cells was evaluated by alizarin red staining. RANK, receptor activator of nuclear factor $\kappa-B$; RANKL, receptor activator of nuclear factor $\kappa$-B ligand; ALP, alkaline phosphatase activity assay; sRANK, recombinant soluble RANK; Mu-RANK, mutant RANK; KD, Knock down.

and $72 \mathrm{~h}$. As presented in Fig. 1A the results demonstrated that sRANK had no significant effect on the proliferation activity of E1 cells, compared with control. The E1 cells were then cultured with $10^{-6}, 10^{-5}$ and $10^{-4} \mathrm{~g} / \mathrm{l}$ RANK protein in osteoblastic differentiation medium for 7 days to measure the ALP activity and then with $10^{-6}, 10^{-5}$, and $10^{-4} \mathrm{~g} / \mathrm{l}$ RANK protein for 2 weeks to detect mineralization by alizarin red staining. The RANK increased the ALP activity in a dose-dependent manner and stimulated mineralization in pre-osteoblastic cells compared with the control group (Fig. 1B and C). Furthermore, the expression of osteocalcin was upregulated in a time-dependent manner (Fig. 1D).

RANK-induces osteoblastogenesis via interaction with $R A N K L$. RANKL is the only RANK-binding protein described in previous reports, therefore the present study investigated if the interaction of RANK with membrane-bound RANKL on osteoblasts leads to osteoblastogenesis. RANKL expression in E1 cells was knocked down by RNAi. The cells transfected with Stealth ${ }^{\mathrm{TM}}$ RNAi oligonucleotide of RANKL for $48 \mathrm{~h}$ demonstrated a significant downregulation in gene expression (Fig. 2A). RANKL knockdown reduced ALP activity in cells treated with $10^{-4} \mathrm{~g} / \mathrm{l} \mathrm{sRANK}$ protein. RANK demonstrated a weaker activity in RANKL-deficient cells compared with wild-type cells (Fig. 2B). These results were verified by alizarin red staining (Fig. 2C).

MAPK p38 signaling pathway is involved in RANK stimulation in El cells. In order to understand the mechanism of the reverse signaling, the present study analyzed the signaling pathways of RANK-induced osteoblastogenesis. Activation of p38 MAPK has been revealed to be required for osteoblast differentiation (11). Addition of RANK protein to the cell culture markedly enhanced phosphorylation of p38 following $10 \mathrm{~min}$ and the phosphorylation was maintained for 240 min (Fig. 3A). The p38 MAPK inhibitor, SB203580, significantly diminished the ALP activity and the size and number of calcified nodules in a dose-dependent manner (Fig. 3B and C).

RANK-RANKL interaction blocks reverse signaling in osteoblast and osteoclast co-culture system. The osteoblast and osteoclast co-culture system is unable to be continuously maintained, therefore only the phosphorylation of p38 MAPK signaling factor was examined, a short period following induction of the co-culture (30, 60, 90 and $120 \mathrm{~min})$.

The results indicated that p38 MAPK in co-culture cells was observed to undergo phosphorylation a short time period following osteoblast and osteoclast interaction (Fig. 4A and B). To examine if the RANKL reverse signal is important in this process, soluble RANKL was added to the co-culture system and mu-RANKL with low activity as a control (Fig. 4C). Abundant soluble RANKL blocked osteoblast contact with osteoclasts via RANKL-RANK binding. The result demonstrated that the phosphorylation may be blocked by soluble RANKL. The soluble RANKL is a highly specific receptor to RANK and the findings suggested that the specific binding interaction RANKL-RANK blocked the reverse signaling pathways from osteoclast to osteoblast.

\section{Discussion}

It has previously been demonstrated that osteoblasts regulate osteoclasts via the RANKL-RANK-OPG signaling pathway and are able to exhibit bidirectional signaling (12). Communication between osteoclasts and osteoblasts affects mass and quality of bone production. Excess remodeling associated with aging reduces bone mass, whereas suppressed bone remodeling increases it. Administration of antiresorptive agents including bisphosphonates increases bone mass in patients with osteoporosis or other remodeling-associated diseases. However, bone strength is determined by bone quality in addition to mass (13). Suppressed bone remodeling reduces bone quality due to accumulation of micro 
A

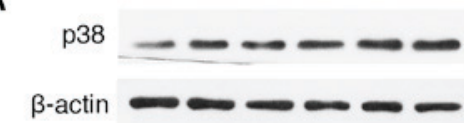

$\begin{array}{llllll}10 & 30 & 60 & 90 & 120 & 240\end{array}(\mathrm{~min})$

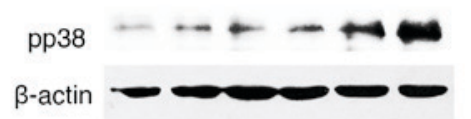

C

$10 \quad 30 \quad 60 \quad 90 \quad 120 \quad 240$ (min)

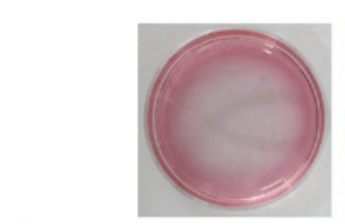

$\mathrm{SB} 203580(\mu \mathrm{M}) \quad-$

$10^{-4} \mathrm{~g} / \mathrm{L}$ Mu-RANK

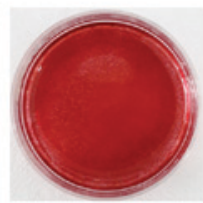

B
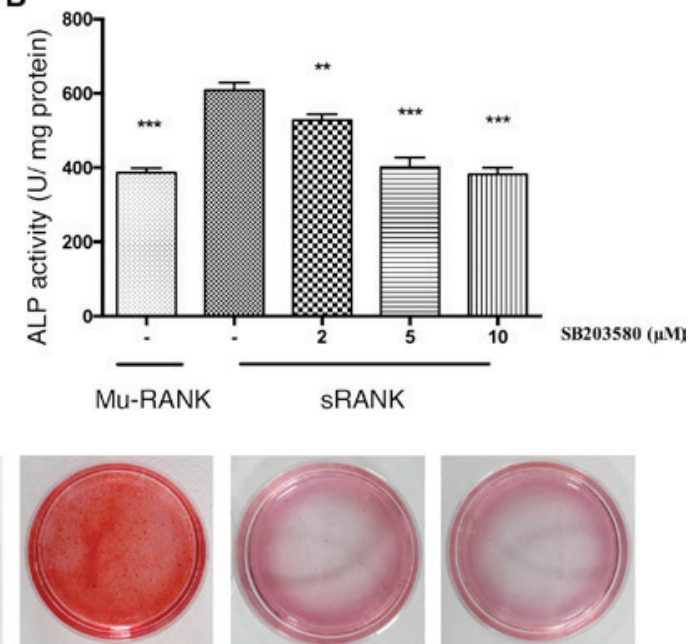

2

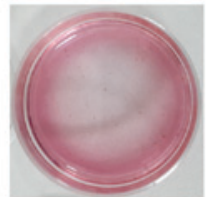

5

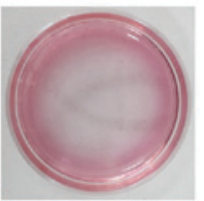

10

Figure 3. Signaling pathways involved in RANK stimulation in E1 cells. Cells were cultured in the presence of $10^{-4} \mathrm{~g} / 1 \mathrm{sRANK}$ or Mu-RANK protein with 0-10 $\mu \mathrm{M}$ p38 inhibitor (SB203580). (A) Post-confluent E1 cells were stimulated with 10 $0^{-4} \mathrm{~g} / 1$ RANK protein for 10-240 min. Phosphorylated p38, total p38 and $\beta$-actin were detected by western blotting. The osteogenic effects of sRANK on E1 cells in absence or presence of SB203580 were measured by (B) ALP activity on day 7 and examined by $(C)$ alizarin red staining on day $14 .{ }^{* * *} \mathrm{P}<0.01{ }^{* * * *} \mathrm{P}<0.001 \mathrm{vs} .10^{-4} \mathrm{~g} / 1$ sRANK protein with no SB203580. RANK, receptor activator of nuclear factor $\mathrm{k}-\mathrm{B}$; ALP, alkaline phosphatase activity assay; sRANK, recombinant soluble RANK; Mu-RANK, mutant RANK; p, phosphorylated.

A

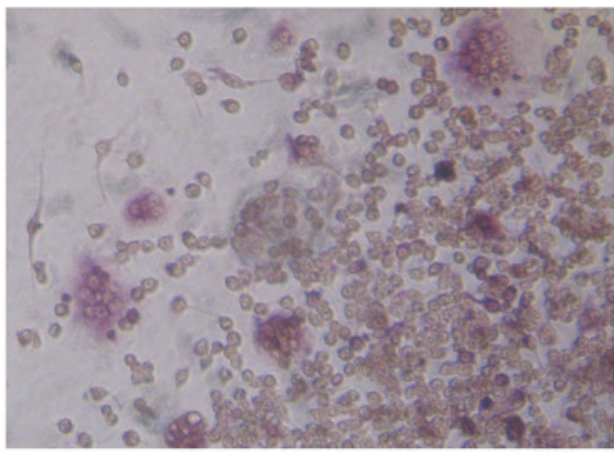

\section{C}

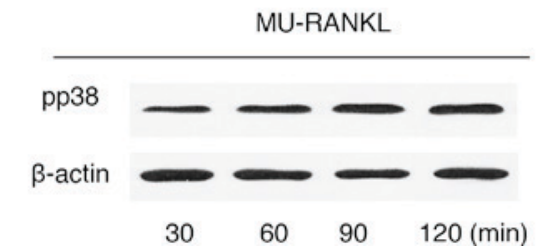

B

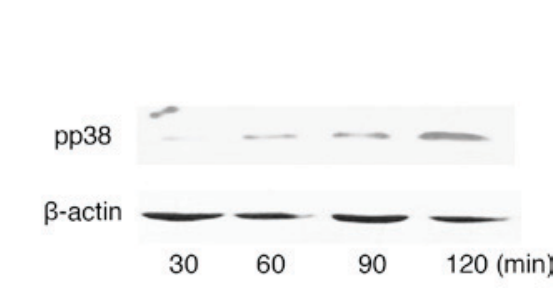

SRANKL

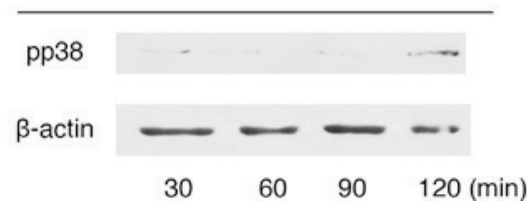

Figure 4. RANK-RANKL interaction in osteoblast and osteoclast co-culture system. (A) Light microscopy images of the osteoblast and osteoclast co-culture following $30 \mathrm{~min}$. (Magnification x200). (B) The phosphorylation of p38 was detected by western blotting within a short time period following co-culture (30, 60, 90 and $120 \mathrm{~min}$ ). (C) A total of $5 \mathrm{nM}$ sRANKL was added to the co-culture system and Mu-RANKL as control. The phosphorylation of p38 was detected by western blotting. RANK, receptor activator of nuclear factor $\kappa-B$; RANKL, receptor activator of nuclear factor $\kappa$-B ligand; sRANKL, recombinant soluble RANKL; Mu-RANKL, mutant RANKL; p, phosphorylated.

fractures and reduced restructuring of bone architecture (14). Therefore, understanding osteoclast-osteoblast communication and bone quality is essential to design effective interventions that will simultaneously prevent bone loss and maintain bone quality.

It has previously been suggested that recombinant murine RANK protein effectively inhibits the activity of osteoclasts and the resulting bone resorption (9), however the exact mechanism remains to be elucidated. To the best of our knowledge, the present study demonstrated for the first time that the soluble RANK stimulated differentiation and mineralization of osteoblastic cells in vitro, which indicated that the single protein upregulated osteoblastogenesis. The effect was achieved via activation of the RANK-RANKL reverse signal. These results 
suggested that RANK exerted an effect on stimulation of bone formation.

RANKL, which is a member of the TNF family, is a type II trans-membrane protein containing a C-terminal TNF homology domain. Numerous TNF family proteins may be expressed in a soluble form or released from the cell surface via specific protease cleavage $(15,16)$, however, the majority act as membrane-bound factors and require direct cell-to-cell contact. The possibility therefore exists for the bidirectional transfer of information upon TNF-TNFR ligation. TNF family member ligands induce forward signals delivered via their respective receptors that promote cell survival, death, differentiation or inflammation in cells expressing members of the TNFR family, depending on the activation state of the cells and the expression levels of the TNFR family molecules. TNFR family members additionally may serve as ligands to initiate reverse signaling, regulation of cell proliferation, cytokine secretion, oxidative burst, class switch and $\mathrm{T}$ cell maturation in cells expressing molecules of the TNF family (4). Previous studies have suggested that reverse signaling occurs between the members of the TNF family receptors, including TNF- $\alpha$, CD-40, Fas and RANK, and their respective membrane-bound ligands, that transmit signals as receptors $(5,17)$. Numerous molecules of the TNF family serve as counter-receptors and transmit reverse signals activated by their respective TNF receptors (18).

Soluble RANK may bind to RANKL and block the RANKL-RANK forward signal to inhibit osteoclastogenesis. However, the present study suggested that soluble RANK bound to trans-membrane RANKL and activated the RANK-RANKL reverse signal to promote osteoblastogenesis, a pathway reversal which has been confirmed to occur in other TNF family members. RANK is an artificial synthetic peptide that binds RANKL, therefore it was hypothesized that it would act as an endogenous ligand to RANKL. The interaction of RANKL and RANK exhibits the potential to transmit a bidirectional signal to activate osteoclasts and osteoblasts via forward and reverse pathways, respectively.

The crystal structure of RANK-RANKL binding interaction was identified. The present study constructed several variations of RANK and RANKL sequences, each with one amino acid mutation introduced. The affinity of RANK and RANKL was measured using a BIAcore 3000 system as previously described (7). Mu-RANK, with one amino acid mutation present in the key site, has been demonstrated to bind RANKL with a one-fifty affinity compared with wild-type RANK (8), indicating the importance of the crystal structure in protein interaction research. To examine if RANK depends on RANKL in its osteoblastogenesis activity, RANKL expression in E1 cells was knocked down using RNAi. It was observed that the activity of RANK was partially blocked in the RANKL-knockdown cells. The difference in activity of RANK between the RANKL-deficient and wild type cells indicated that RANKL was involved in RANK-induced osteoblastogenesis. The results suggested that RANK interacted with the RANKL present on the osteoblast and stimulated osteogenic growth via RANKL signaling.

Consistent with a previous report that activation of $\mathrm{p} 38$ MAPK was involved in RANKL signaling in T cells (5), it was demonstrated that the addition of RANK activated p38 MAPK in osteoblastic cells following $30 \mathrm{~min}$, indicating the signal was directly transmitted via RANKL on the cells. OPG and RANK have been demonstrated to bind RANKL. Previous studies demonstrated that both osteoclastogenesis and osteoblastogenesis were enhanced in OPG-deficient mice $(19,20)$, suggesting that bidirectional signaling was enhanced without OPG, as OPG is a decoy receptor for RANKL. The observation of a similar phenotype in RANKL-deficient mice and RANK-deficient mice verifies that RANKL is the only ligand of RANK $(21,22)$.

Co-cultures are of primary interest for in vitro studies as they reconstruct natural conditions and allow elucidation of various aspects of the complex interactions between bone-building and bone-resorbing cells. Successful culture studies involving osteoblast cell lines or primary osteoblasts in combination with peripheral blood mononuclear cells or isolated monocytes have been reported. The cells are of murine or human origin and cultivated with or without the addition of macrophage colony-stimulation factor and RANKL $(23,24)$.

In conclusion, it was demonstrated that RANKL present on osteoblasts exhibited a reverse signal transduction ability. Dysfunction of the reverse signaling pathway may act as an underlying mechanism of osteoporosis. The findings may aid identification of the mechanism regulating the coupling between bone formation and resorption. A reverse signaling via RANKL is involved in part in RANK-induced osteoblast differentiation. The RANK-RANKL reverse signaling interaction may act as a novel and potential mechanism to explain and fully elucidate osteoclast regulation of osteoblasts and provide a novel perspective for the treatment of osteoporosis. RANK-RANKL reverse signaling was studied in the present study and other possible reverse signaling interactions require investigation in future studies.

\section{Acknowledgements}

The present study was supported by the National Nature Science Foundation of China (grant no. 81300711).

\section{References}

1. Boyce BF and Xing L: Biology of RANK, RANKL, and osteoprotegerin. Arthritis Res Ther 9 (Suppl 1): S1, 2007.

2. Li X, Liu Y, Wu B, Dong Z, Wang Y, Lu J, Shi P, Bai W and Wang Z: Potential role of the OPG/RANK/RANKL axis in prostate cancer invasion and bone metastasis. Oncol Rep 32: 2605-2611, 2014

3. Cummings SR, San Martin J, McClung MR, Siris ES, Eastell R, Reid IR, Delmas P, Zoog HB, Austin M, Wang A, et al: Denosumab for prevention of fractures in postmenopausal women with osteoporosis. N Engl J Med 361: 756-765, 2009.

4. Sun M and Fink PJ: A new class of reverse signaling costimulators belongs to the TNF family. J Immunol 179: 4307-4312, 2007.

5. Chen NJ, Huang MW and Hsieh SL: Enhanced secretion of IFN-gamma by activated Th1 cells occurs via reverse signaling through TNF-related activation-induced cytokine. J Immunol 166: 270-276, 2001.

6. Secchiero P, Corallini F, Barbarotto E, Melloni E, di Iasio MG, Tiribelli $\mathrm{M}$ and Zauli G: Role of the RANKL/RANK system in the induction of interleukin-8 (IL-8) in B chronic lymphocytic leukemia (B-CLL) cells. J Cell Physiol 207: 158-164, 2006.

7. Zhang S, Liu C, Huang P, Zhou S, Ren J, Kitamura Y, Tang P, $\mathrm{Bi} Z$ and Gao B: The affinity of human RANK binding to its ligand RANKL. Arch Biochem Biophys 487: 49-53, 2009. 
8. Liu C, Walter TS, Huang P, Zhang S, Zhu X, Wu Y, Wedderburn LR, Tang P, Owens RJ, Stuart DI, et al: Structural and functional insights of RANKL-RANK interaction and signaling. J Immunol 184: 6910-6919, 2010.

9. Zhang J, Zhang L, Xu M, Zhang L, Tang P and Jiang C: Murine RANK protein's inhibition of bone resorption. J Craniofac Surg 22: 2084-2089, 2011.

10. Livak KJ and Schmittgen TD: Analysis of relative gene expression data using real-time quantitative PCR and the 2(-Delta Delta C(T)) method. Methods 25: 402-408, 2001.

11. Hu Y, Chan E, Wang SX and Li B: Activation of p38 mitogen-activated protein kinase is required for osteoblast differentiation. Endocrinology 144: 2068-2074, 2003.

12. Zhao C, Irie N, Takada Y, Shimoda K, Miyamoto T, Nishiwaki T, Suda T and Matsuo K: Bidirectional ephrinB2-EphB4 signaling controls bone homeostasis. Cell Metab 4: 111-121, 2006.

13. Seeman E: Bone quality: The material and structural basis of bone strength. J Bone Miner Metab 26: 1-8, 2008.

14. Mashiba T, Turner CH, Hirano T, Forwood MR, Jacob DS, Johnston CC and Burr DB: Effects of high-dose etidronate treatment on microdamage accumulation and biomechanical properties in beagle bone before occurrence of spontaneous fractures. Bone 29: 271-278, 2001.

15. Aggarwal BB: Signalling pathways of the TNF superfamily: A double-edged sword. Nat Rev Immunol 3: 745-756, 2003.

16. Matthies KM, Newman JL, Hodzic A and Wingett DG: Differential regulation of soluble and membrane CD40L proteins in T cells. Cell Immunol 241: 47-58, 2006.

17. Watts AD, Hunt NH, Wanigasekara Y, Bloomfield G, Wallach D, Roufogalis BD and Chaudhri G: A casein kinase I motif present in the cytoplasmic domain of members of the tumour necrosis factor ligand family is implicated in 'reverse signalling'. EMBO J 18: 2119-2126, 1999.
18. Kemball CC, Lee ED, Szomolanyi-Tsuda E, Pearson TC, Larsen CP and Lukacher AE: Costimulation requirements for antiviral CD8+ T cells differ for acute and persistent phases of polyoma virus infection. J Immunol 176: 1814-1824, 2006.

19. Nakamura M, Udagawa N, Matsuura S, Mogi M, Nakamura H, Horiuchi H, Saito N, Hiraoka BY, Kobayashi Y, Takaoka K, et al: Osteoprotegerin regulates bone formation through a coupling mechanism with bone resorption. Endocrinology 144: 5441-5449, 2003.

20. Mizuno A, Amizuka N, Irie K, Murakami A, Fujise N, Kanno T, Sato Y, Nakagawa N, Yasuda H, Mochizuki S, et al: Severe osteoporosis in mice lacking osteoclastogenesis inhibitory factor/osteoprotegerin. Biochem Biophys Res Commun 247: 610-615, 1998.

21. Kong YY, Yoshida H, Sarosi I, Tan HL, Timms E, Capparelli C, Morony S, Oliveira-dos-Santos AJ, Van G, Itie A, et al: OPGL is a key regulator of osteoclastogenesis, lymphocyte development and lymph-node organogenesis. Nature 397: 315-323, 1999.

22. Dougall WC, Glaccum M, Charrier K, Rohrbach K, Brasel K, DeSmedt T,Daro E,Smith J,Tometsko ME, Maliszewski CR, et al: RANK is essential for osteoclast and lymph node development. Genes Dev 13: 2412-2424, 1999.

23. Bernhardt A, Thieme S, Domaschke H, Springer A Rösen-Wolff A and Gelinsky M: Crosstalk of osteoblast and osteoclast precursors on mineralized collagen-towards an in vitro model for bone remodeling. J Biomed Mater Res A 95: 848-856, 2010

24. Bloemen V, de Vries TJ, Schoenmaker T and Everts V: Intercellular adhesion molecule-1 clusters during osteoclastogenesis. Biochem Biophys Res Commun 385: 640-645, 2009. 